\title{
Crystal structure of a signal recognition particle $A l u$ domain in the elongation arrest conformation
}

\author{
LUC BOUSSET, ${ }^{1,3}$ CAMILLE MARY, ${ }^{2,4}$ MARK A. BROOKS, ${ }^{1,5}$ ANNE SCHERRER, ${ }^{2}$ KATHARINA STRUB, ${ }^{2}$ \\ and STEPHEN CUSACK ${ }^{1}$ \\ ${ }^{1}$ European Molecular Biology Laboratory, Grenoble Outstation, 38042 Grenoble Cedex 9, France \\ ${ }^{2}$ Département de Biologie Cellulaire, Université de Genève, Sciences III, 1211 Geneva 4, Switzerland
}

\begin{abstract}
The signal recognition particle (SRP) is a conserved ribonucleoprotein particle that targets membrane and secreted proteins to translocation channels in membranes. In eukaryotes, the Alu domain, which comprises the $5^{\prime}$ and $3^{\prime}$ extremities of the SRP RNA bound to the SRP9/14 heterodimer, is thought to interact with the ribosome to pause translation elongation during membrane docking. We present the $3.2 \AA$ resolution crystal structure of a chimeric Alu domain, comprising Alu RNA from the archaeon Pyrococcus horikoshii bound to the human Alu binding proteins SRP9/14. The structure reveals how intricate tertiary interactions stabilize the RNA $5^{\prime}$ domain structure and how an extra, archaeal-specific, terminal stem helps constrain the Alu RNA into the active closed conformation. In this conformation, highly conserved noncanonical base pairs allow unusually tight side-by-side packing of $5^{\prime}$ and $3^{\prime}$ RNA stems within the SRP9/14 RNA binding surface. The biological relevance of this structure is confirmed by showing that a reconstituted full-length chimeric archaeal-human SRP is competent to elicit elongation arrest in vitro. The structure will be useful in refining our understanding of how the SRP Alu domain interacts with the ribosome.
\end{abstract}

Keywords: signal recognition particle; translation; X-ray crystallography; RNA; RNA folding

\section{INTRODUCTION}

In eukaryotic cells, secretory and membrane proteins are targeted to the endoplasmic reticulum (ER) by the universally conserved ribonucleoprotein particle SRP and its membrane-associated receptor (SRP receptor [SR] or docking protein) (for review, see Saraogi and Shan 2011; Nyathi et al. 2013). A nascent polypeptide chain emerging from a translating ribosome with an N-terminal hydrophobic signal sequence, the hallmark of ER-targeted proteins, is recognized by the SRP54 component of SRP and targeted to the ER via the interaction of SRP and SR. This process is controlled by GTP-binding and hydrolysis by SRP54 and SR leading to the transfer of the nascent chain to the translocation channel. The protein then integrates into or transfers across the membrane in a cotranslational manner. During targeting, the mammalian SRP delays nascent chain elongation, which en-

\footnotetext{
${ }^{3}$ Present address: Laboratoire d'Enzymologie et Biologie Structurale, UPR 3082 CNRS, 91198 Gif-sur-Yvette Cedex, France

${ }^{4}$ Present address: Department of Human Protein Sciences, Faculty of Medicine, University of Geneva, 1211 Geneva 4, Switzerland

${ }^{5}$ Present address: Evotec (UK) Ltd, Abingdon, OX14 4SA, UK

Abbreviations: SRP, signal recognition particle; MAD, multiwavelength anomalous dispersion; RNP, ribonucleoprotein particle

Corresponding author: cusack@embl.fr

Article published online ahead of print. Article and publication date are at http://www.rnajournal.org/cgi/doi/10.1261/rna.047209.114.
}

sures the efficient delivery of the nascent chain to the ER in human cells (Lakkaraju et al. 2008).

Eukaryotic SRPs possess a composite structure, comprising the universally conserved S-domain and the Alu domain that is found in eukaryotes, archaea, and some bacteria (Walter and Blobel 1980). Human SRP exemplifies a typical eukaryotic particle and consists of six proteins (Walter and Blobel 1980), which bind to the functionally essential 7SL RNA (human SRP RNA) of $300 \mathrm{nt}$. The signal recognition and targeting functions are assigned to the S-domain; whereas, the Alu domain harbors the elongation arrest function (Siegel and Walter 1988). The Alu domain includes the $5^{\prime}$ and $3^{\prime}$ extremities of 7SL RNA, forming the Alu RNA, bound to the SRP9/14 heterodimer (Fig. 1A). Our previous structural studies revealed that human SRP9 and SRP14 are structurally homologous (Birse et al. 1997) and together form a six-stranded $\beta$ sheet with a concave surface which is the major binding site for Alu RNA (Weichenrieder et al. 2000).

In most $A l u$ domains, the $5^{\prime}$ and $3^{\prime}$ portions are linked by a single-stranded hinge region (Fig. 1A,B), but are otherwise mobile with respect to each other (Weichenrieder et al.

\footnotetext{
(C) 2014 Bousset et al. This article is distributed exclusively by the RNA Society for the first 12 months after the full-issue publication date (see $\mathrm{http} / / /$ rnajournal.cshlp.org/site/misc/terms.xhtml). After 12 months, it is available under a Creative Commons License (Attribution-NonCommercial 4.0 International), as described at http://creativecommons.org/licenses/ by-nc/4.0/
} 
A

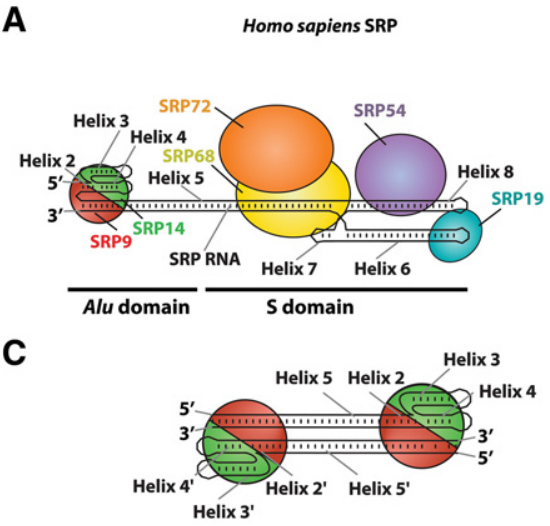

$\mathbf{E}$

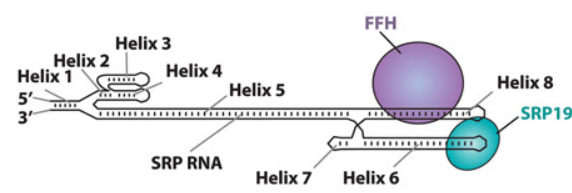

B

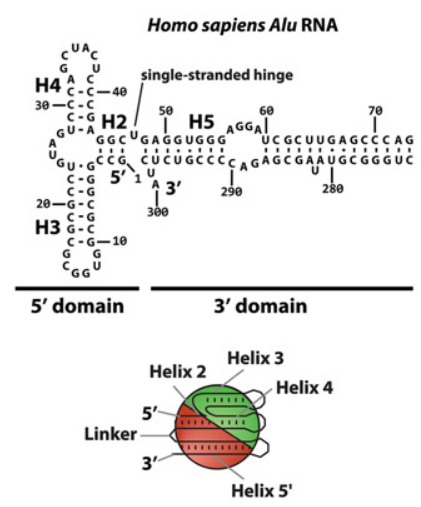

$\mathbf{F}$

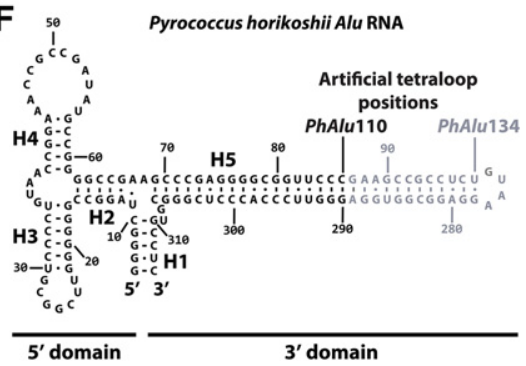

FIGURE 1. The human and archaeal SRP and Alu domains. (A) Schematic representation of the human SRP showing location of the six proteins (four bound to the $S$ domain and two bound to the Alu domain) and the eight helical segments of the 7SL RNA numbered according to the standard nomenclature of SRP RNAs (Zwieb et al. 2005). SRP proteins are colored as follows: SRP9, red; SRP14, green; SRP68, yellow; SRP72, orange; SRP19, cyan; SRP54/FFH, purple. (B) Sequence of the human $A l u$ RNA with RNA helices marked. Note the single-stranded hinge between the $5^{\prime}$ and $3^{\prime}$ domains. $(C)$ Schematic diagram of the domain-swapped dimeric crystal structure of a rigidly linked human Alu RNA variant (SA86) bound to human SRP9/14 (PDB code 1e8s) (Weichenrieder et al. 2000). Each SRP9/14 heterodimer binds two separate RNA molecules. (D) Hypothetical model of the physiologically active, closed state of the human Alu domain. The single-stranded hinge between the $5^{\prime}$ and $3^{\prime}$ domains allows a single $A l u$ RNA molecule to occupy both binding sites on the SRP9/14 heterodimer (Weichenrieder et al. 2000). (E) Schematic representation of the Pyrococcus horikoshii SRP showing only two proteins bound to the S domain and the additional Helix 1 formed by complementary extensions to the $5^{\prime}$ and $3^{\prime}$ termini. Nomenclature and coloring as in A. The positions of the tetraloops added to close the PhAlu110 and PhAlu134 RNAs are indicated. (F) Sequence of the P. horikoshii Alu RNA with RNA helices marked. Note the threeway junction formed from helices 1,2, and 5 which rigidifies this region.

2001). In previous work, we only succeeded in crystallizing this structure by restricting its flexibility, which was done by producing an artificial, circularly permuted $A l u$ RNA with a rigid linkage (Weichenrieder et al. 2000, 2001). The resulting Alu domain structure was a domain-swapped dimer with each RNA in the extended, "open" conformation and each SRP9/ 14 heterodimer bound to two sites, consistent with those mapped by hydroxyl radical cleavage experiments (Strub et al. 1991), but on different adjacent RNA molecules (Fig. 1C; Weichenrieder et al. 2000). The likely monomeric physiological structure was inferred from the crystallographic model by proposing that the Alu RNA must fold back on to itself such that one SRP9/14 heterodimer interacts with a single RNA molecule, in a "closed" conformation (Fig. 1D; Weichenrieder et al. 2000, 2001). This model was later confirmed by modeling into the low-resolution cryo-electron microscopy map of the entire mammalian SRP-ribosome complex (Halic et al.

2004). However, a high-resolution structure of the physiological, closed form of the Alu domain is still lacking.

The elongation arrest activity of the Alu domain primarily involves SRP9/14. Cellfree translation and translocation assays and in vivo studies in human cells revealed a stretch of basic amino acid residues in SRP9 and at the C-terminus of SRP14 to be essential for elongation arrest activity (Thomas et al. 1997; Lakkaraju et al. 2008; Mary et al. 2010). To better understand how the SRP Alu domain interacts with the ribosome and to elucidate the mechanism of elongation arrest, a more precise model of the closed conformation Alu domain structure is required. As the human Alu domain complex was resistant to crystallization, we switched to work with the Alu domain of the archaeon Pyrococcus horikoshii. Archaeal SRP RNAs possess both Alu and S-domains, but protein homologues of only SRP19 and SRP54 have been identified, and very little is known about protein translocation in archaeal species (Fig. 1E; Zwieb and Bhuiyan 2010). Archaeal SRP RNAs have extra sequences at their $5^{\prime}$ and $3^{\prime}$ ends, which are predicted to form an additional helix ( $\mathrm{H} 1)$ in the Alu domain (Fig. 1E; Larsen and Zwieb 1991). This would create a threeway junction comprising $\mathrm{H} 1, \mathrm{H} 2$, and $\mathrm{H} 5$ (Fig. 1F), which we hypothesized might stabilize the closed conformation of the archaeal Alu domain. A similar structure is also predicted to exist in certain eubacteria which possess an $A l u$ domain, such as Bacillus subtilis (Poritz et al. 1988).

Here, we describe the crystal structure of a chimeric Alu domain comprising the P. horikoshii Alu RNA (PhAlu) in complex with human SRP9/14. In this structure, the overall fold of the archaeal Alu domain RNA and in particular the conserved tertiary contacts between the $5^{\prime}$ and $3^{\prime}$ domains are revealed in detail. Furthermore, we demonstrate that a reconstituted full-length chimeric SRP is active in elongation arrest assays suggesting that the new structure will be a robust model for the human Alu domain.

\section{RESULTS}

\section{Structure determination of the $\boldsymbol{P}$. horikoshii Alu domain with human SRP9/14}

For structure determination, two PhAlu domain RNAs, PhAlu110 (110 nt) and PhAlu134 (134 nt) which include 
different lengths of helix 5 closed by an artificial tetraloop were designed (Fig. 1F). These were synthesised by in vitro transcription and shown to form stable 1:1 complexes with human SRP9/14 (h9/14) by gel filtration (Supplemental Fig. 1). Crystallization trials of both RNAs alone and in complex with h9/14 were performed. However, diffracting crystals were only obtained of the RNP in each case. The structure of PhAlu134-h9/14 was solved by the MAD method using seleno-methionine labeled protein and refined to $3.20 \AA$ resolution, with one complex per asymmetric unit. The structure of PhAlu110-h9/14 structure was subsequently solved by molecular replacement at $3.35 \AA$ resolution and contains two complexes in the asymmetric unit. Most of the RNA and protein is well defined in the electron density (Supplemental Fig. 2) apart from the extra, distal section of helix 5 in PhAlu134, the C-terminal extremities of h9 (beyond Met83) and h14 (beyond Lys96) and the internal loop of h14 (43-49 have poor electron density). All three independent examples of the core of the PhAlu-h9/14 RNP are very similar (Supplemental Fig. 3) and so only the higher resolution PhAlu134-h9/14 structure is described in detail. Crystallographic details are given in Supplemental Tables 1 and 2.

\section{Structural features of the $P$. horikoshii Alu RNA $5^{\prime}$ domain}

The PhAlu domain RNAs are of approximate dimensions $112 \times 38 \times 30 \AA($ PhAlu134) and $87 \times 38 \times 30 \AA$ (PhAlu110). The overall RNA fold resembles a shepherd's crook, with the $3^{\prime}$ domain (helix H5) representing the staff and the $5^{\prime}$ domain its hook (Fig. 2A,B). As in the human Alu RNA, the PhAlu $5^{\prime}$ domain (stems 2,3 , and 4 ) forms a three-way $\tau$-junction in which stems 2 and 4 are coaxially stacked and connected by a U-turn to stem 3 (Weichenrieder et al. 2000). The corresponding hairpin loops L3 and L4 interact through extensive base-pairing (Fig. 2A). Both $\tau$-junction and helix 5 are accommodated within the concave $\beta$-sheet surface of h9/14 as predicted in the model for the active conformation of the human Alu domain.

The PhAlu RNA structure is crowned by a three-way junction formed by helices $\mathrm{H} 1, \mathrm{H} 2$, and $\mathrm{H} 5$ (hereafter referred to as $\mathrm{J} 1 / 2 / 5$ ), which has the form of an inverted tuning fork (Fig. 2A, C). Helix 1 comprises five base pairs from the $5^{\prime}$ and $3^{\prime}$ extremities of the PhAlu RNA and forms the fork handle. The junction orientates the two arms of the tuning fork, helix $\mathrm{H} 2$, and helix $\mathrm{H} 5$ into a quasiparallel orientation as required for binding to SRP9/14. A buckled base triple, formed by A68 interacting with the minor groove of the G13.C65 base pair, plays a central role in the three-way junction. The inflexible J1/2/5 junction constrains the RNA to be in the active conformation, rigidifies the junction and likely prevents rotation of helix 5 relative to the $5^{\prime}$ domain, a movement that was postulated to occur during assembly of the human Alu RNA in which the $5^{\prime}$ and $3^{\prime}$ ends are not base-paired (Weichenrieder et al. 2000, 2001).
Loops L3 and L4, which in PhAlu are, respectively, 7 and $11 \mathrm{nt}$ long, are stabilised by interloop base pairs and additional buttressing interactions. Five G:C Watson-Crick base pairs between nucleotides 25-29 (CGGCG) and 52-48 (GCCGC) create a robust double helix that runs perpendicular to stems 3 and 4 (Fig. 2A,B). The G29.C48 end of this helix is extended by an additional noncanonical base pair between U30 and A46, C47 being flipped out. Sharp kinks in the RNA backbone at C48 and A53 of one strand and C25 of the other, bring neighboring nucleotides back behind the interacting loops such that stabilizing base triples and additional hydrogen bonds are formed. The flanking bases 22-GUU of loop 3 stack onto each other but are not base-paired. G22 makes a single hydrogen bond with U30 and U24 makes a U-turn interaction (hydrogen bond via N3) with the phosphate of G27, buttressing this region of the structure. Several unpaired nucleotides in L4 are involved in base triples (Fig. 2D). A45 stacks on the noncanonical G57:A44 that forms the last base pair of stem 4 and makes a buckled triplet with the interloop Watson-Crick G29-C48 base pair. A55 binds to the minor groove of the loop-loop base pair G26.C51 and represents an archetypal A-minor motif (Nissen et al. 2001). A53, which stacks on A55, forms hydrogen bonds with the base pair C25.G52 via its Hoogsteen edge in a similar manner as seen in the A platform (Cate et al. 1996) and in the A-A sidestep (Conn et al. 1999) motifs. The intervening base U54 is flipped out, like U38 in the human Alu RNA domain structure and is completely exposed to solvent. The multiple canonical and noncanonical interactions between L3 and L4 create a particularly intricate and stable structure. Compared with the human Alu $5^{\prime}$ domain structure, where there are only three interloop base pairs and no base triplets, the extra structural buttressing of the PhAlu $5^{\prime}$ domain probably reflects the need for higher thermal stability in thermophilic organisms.

\section{Stem 5 structure and tertiary interactions between the $5^{\prime}$ and $3^{\prime}$ domains}

The PhAlu134 RNA comprises 30 base pairs (roughly half) of helix $\mathrm{H} 5$ and is capped by an artificial tetraloop. H5 is $\sim 90 \AA$ in length and slightly bent (Fig. 2A,B). Superposition of the PhAlu110 and PhAlu134 RNAs shows that despite the high degree of similarity of the two RNAs, the direction of helix 5 diverges slightly after the 3 tertiary interaction (Supplemental Fig. 3). This may be due to crystal packing but likely reflects flexibility in the structure at this point, which is approximately where bending of SRP RNA (hinge 2) was observed in a cryoEM structure of mammalian SRP bound to the ribosome (Halic et al. 2004). The majority of helix H5 base pairs are Watson-Crick, but there are also three regions with noncanonical base pairs (boxed in Fig. 2A). Of these, the most significant are G78.A298 and C79.C297, which are at a position analogous to the first asymmetric internal loop found in helix H5 of human SRP RNA (Fig. 1B). The PhAlu RNA structure 

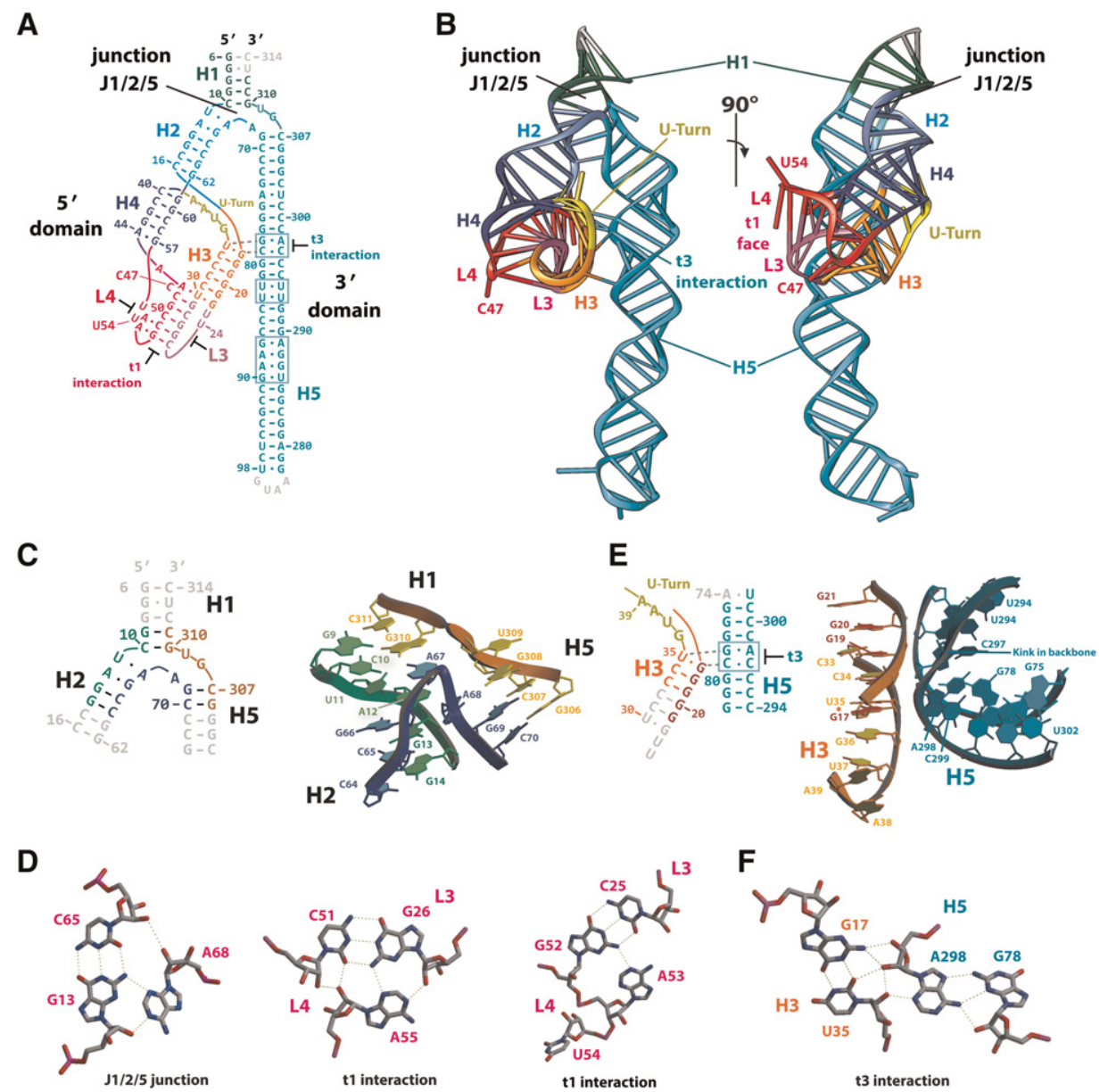

FIGURE 2. Structure of the PhAlu134 RNA. (A) Structure based secondary structure of the PhAlu134 RNA. SRP secondary and tertiary structural elements are labeled as recommended (Zwieb et al. 2005) as follows: helix (H), loop (L), tertiary interaction (t). Color-coding used is as follows: H1 (green), H2 (light blue), H3 (orange), H4 (blue), H5 (cyan), extra 3' nucleotides and tetraloop (gray). Base pairs are depicted as Watson-Crick (dash), others (dot). Numbering is according to full-length P. horikoshii SRP RNA (http://rnp.uthscsa.edu/rnp/SRPDB/rna/srprnaphylolist_arch.html). (B) Cartoon representation of the structure of the PhAlu134 SRP RNA with SRP9/14 proteins removed for clarity. Color-coding and labeling used are as in A. At the surface of the tertiary interaction t1, a flat "face" is formed by base triples, and C47 and U54 are flipped out into the solvent. (C) Details of the three-way junction between H1, H2, and H5 (J1/2/5). (Left) Schematic of the junction. Residues G9-G14 (from helix 1 to 2) are shown in green, C64-C70 (helices 2-5) are shown in blue, and residues G306-C311 (helices 5-1) are in orange. (Right) The RNA backbone (ribbon) and bases are color-coded as on the left. Interactions around A67 and A68 stabilize the junction, whereas these bases would form a flexible hinge in the human counterpart structure. (D) Stabilization of the structure by base triples shown in stick representation. (Left) G13-C65-A68 base triple in the $\mathrm{J} 1 / 2 / 5$ junction. (Center and right) Base triples reinforce the L3-L4 tertiary interaction (t1). Interloop base pairs C51.G26 and G52.C25 form A-minor motif base triples with A55 and A53, respectively. (E) The t 3 tertiary interaction between the $5^{\prime}$ and $3^{\prime}$ domains. (Left) Schematic of the t3 stem-stem interaction drawn as in C. (Right) The H5 backbone is distorted by noncanonical base pairs to permit close packing of the two stems. $(F)$ The universally conserved G17.U35 wobble base pair of helix 3 interacts with A298 of helix 5. A298 and G78 interact in turn via a sheared base pair at a position that in many other species is an asymmetric internal loop of helix 5.

confirms that this is the major site of contact between the $5^{\prime}$ and $3^{\prime}$ domains, as previously deduced from the low-resolution human Alu domain structure (Weichenrieder et al. 2000). Now the significantly improved resolution permits the precise nature of the interdomain contacts to be determined. Stem 3 of PhAlu RNA contains a conserved G17.U35 wobble base pair that is strictly conserved in most Alu RNAs (e.g., the human counterpart is G4.U23) (Fig. 1B). This base pair mediates the interaction of the $5^{\prime}$ domain with the noncanonical G78.A298 and C79.C297 base pairs resulting in a local distortion of the helix 5 structure (Fig. 2E).
The nitrogen N2 of G17 is exposed at the surface of the minor groove of helix 3 and forms a network of hydrogen bonds with the $2^{\prime}$ and $3^{\prime}$ ribose oxygen atoms as well as N3 of the base of A298 of helix 5 as another A-minor motif (Doherty et al. 2001). Both $\mathrm{O} 2$ and $\mathrm{O} 2^{\prime}$ of $\mathrm{U} 35$ make additional hydrogen bonds with O2' of A298. The consequence of this motif is to distort helix 5, flattening the curvature of the double helix and allowing close contact of the two stems (Fig. 2E). Interstem contacts are also made from residues G17-G19 and U34 of the $5^{\prime}$ domain, to atoms of C297 and C299 of the $3^{\prime}$ domain. We presume that a very similar stem-stem 
contact can be made by the human Alu RNA with the equivalent G4.U23 wobble base pair interacting with noncanonical base pairs of the first asymmetric loop (notably G57:A289), as suggested by the low resolution human Alu domain structure (Weichenrieder et al. 2000). Therefore, we propose to extend the nomenclature of SRP RNAs (Zwieb et al. 2005) to take into account this additional conserved contact and suggest to refer to this tertiary interaction as "t3" (Fig. 2A).

\section{hSRP9/14 binding to PhAlu RNA}

Despite the evolutionary distance between the two organisms, it is remarkable that the hSRP9/14 proteins bind to the PhAlu RNA in an essentially identical manner to observed previously in the human Alu domain structures (Fig. 3A; Weichenrieder et al. 2000). Indeed, superposition of the PhAlu RNA structure with the derived model of the active, closed form of the human Alu domain RNA (Fig. 3B) shows that the conformation of the RNA core of the Alu domain (both $5^{\prime}$ and $3^{\prime}$ domains) is well-predicted by our previous model (Fig. 1D) and strikingly well-conserved between very distantly related species. Fifty-three bases can be structurally superposed in this way, of which 26 are identical, with an RMSD of the phosphate-ribose backbone atoms of $1.77 \AA$. The similarity of the RNA structure explains why the detailed protein-RNA interactions are also highly conserved. The $\beta$-sheet of hSRP9/14 contacts both the U-turn and the neighboring helices $\mathrm{H} 3$ and $\mathrm{H} 4$, with basic residues from hSRP14 providing the majority of these contacts, as previously described (Weichenrieder et al. 2000). Interestingly, the internal loop of hSRP14 (residues 34-54), which was disordered in previous human $A l u$ domain structures (i.e., in the absence of stem 5), is partially visible when bound to $P h A l u$ RNA. It extends in the direction of the J1/2/5 junction with contacts to the U-turn nucleotide A38 and C65 of stem 2 (Fig. 3C). Specifically, Arg36 interacts with the phosphate of A38, the main chain of residues 37-39 stack on the base of A38 and Lys 38 interacts with the phosphate of C65. These observations are consistent with previous work showing that the $\mathrm{N}$-terminal part of the loop was the most critical for RNA binding (Bui et al. 1997). RNA interaction.
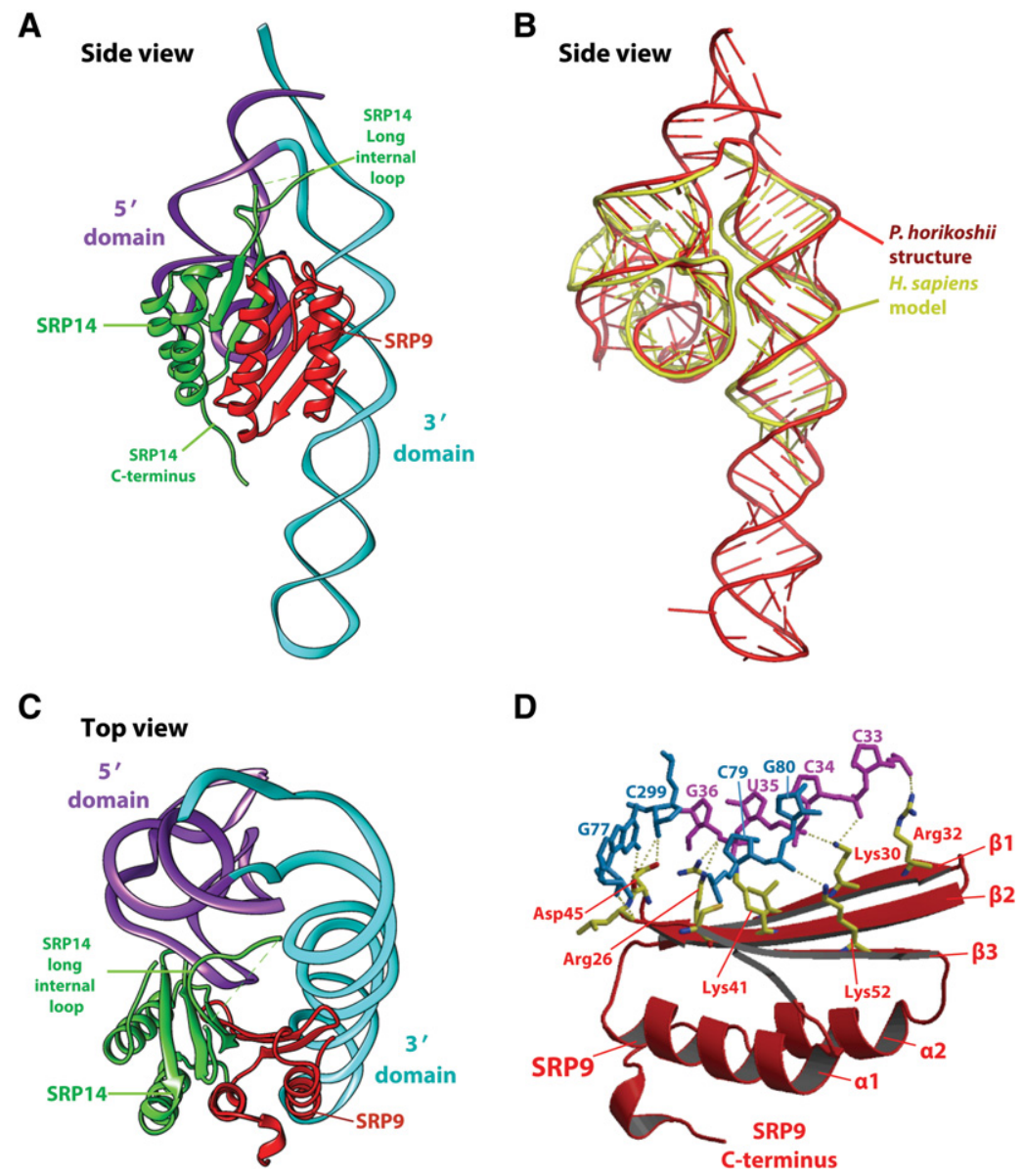

FIGURE 3. SRP9/14 binding to PhAlu SRP RNA. (A) Overall structure of the chimeric Alu domain RNP. The backbone of PhAlu134 RNA is drawn as a ribbon and colored purple and cyan for the $5^{\prime}$ and $3^{\prime}$ domains, respectively. The hSRP9 and hSRP14 are drawn as, respectively, red and green cartoons. SRP14 contacts exclusively the $5^{\prime}$ and $3^{\prime}$ domain whereas SRP9 contacts both RNA domains. The long internal loop, which extends toward the J1/2/5 junction, is shown dotted where poorly visible. The basic C-terminal extension of SRP14 extends toward the Sdomain. (B) Comparison of PhAlu134 RNA with the human Alu RNA structure model. A structural superposition of the human Alu domain model, derived from the structure of a circularly permutated RNA (Weichenrieder et al. 2000) (yellow backbone with bases as sticks) onto PhAlu134 RNA (red) highlights the highly conserved tertiary fold of the Alu RNA core from divergent organisms. Superposing just the 5' domain (nucleotides 15-65 in PhAlu and 2-47 in human Alu RNA) allows matching of $31 \mathrm{nt}$ (19 identities) with an RMSD of $1.47 \AA$. For the $5^{\prime}$ and $3^{\prime}$ domain taken together, 53 bases can be matched (26 identities) with an RMSD of $1.77 \AA$. (C) Diagram showing how both the $5^{\prime}$ domain and $3^{\prime}$ stem of PhAlu134 RNA are accommodated side by side within the concave $\beta$-sheet surface of SRP9/14. (D) Protein-RNA interactions mediated by SRP9 at the 3 tertiary interaction. SRP9 (red with yellow side chains) interacts with nucleotides from both the PhAlu $5^{\prime}$ (purple) and $3^{\prime}$ (cyan) domains thus reinforcing the t 3 tertiary

Jointly, SRP9/14 bury $\sim 1280 \AA^{2}$ of the surface area of the RNA, of which about two-thirds is by SRP14. Whereas SRP14 contacts only the $5^{\prime}$ domain, the contiguous $\beta$-sheet of SRP9 interacts equally with the $5^{\prime}$ and $3^{\prime}$ domains, clasping them together (Fig. 3C). While SRP9 strand $\beta 1$ interacts with residues of the U-turn in the $5^{\prime}$ domain, strands $\beta 2$ and $\beta 3$ contact the minor groove of stem 5, as do residues Asp45 to Cys48 of the $\beta 2-\beta 3$ loop (Fig. 3D). A network of hydrogen bonds is central to this interaction, including an interaction of the carbonyl group of Leu46 with the $2^{\prime}$ hydroxyl group 
of G77. The side-chain of Lys52 is also engaged in hydrogen bonds to the phosphate G80 of helix 5. Interestingly, these SRP9-RNA contacts colocalize with the t 3 tertiary RNA interaction, adding further stabilization.

\section{Functional replacement of the human Alu domain by the $P$. horikoshii Alu domain}

We next investigated whether the PhAlu domain could function in a similar manner to 7SL Alu domain. For this purpose, we constructed the PAHS RNA comprising the PhAlu 5' domain (including a short stretch of nucleotides from the $3^{\prime}$ end) fused to the S-domain of human 7SL RNA (Supplemental Fig. 4A). We first examined whether such a chimeric SRP RNA was functionally active. Denaturing and native PAGE was used to compare the integrity and heterogeneity of human full-length synthetic 7SL (7SLC) (Mary et al. 2010) and PAHS RNAs, respectively (Fig. 4A). In addition to some heterogeneity observed for both RNAs in native PAGE, PAHS RNA migrated more slowly than 7SLC RNA in a distinct major band consistent with its larger size. 7SLC and PAHS RNAs were reconstituted into SRP using recombinant and native SRP proteins (Huck et al. 2004).

Reconstitution reactions (RCs) were directly added to wheat-germ lysate programmed for translation with prepro-
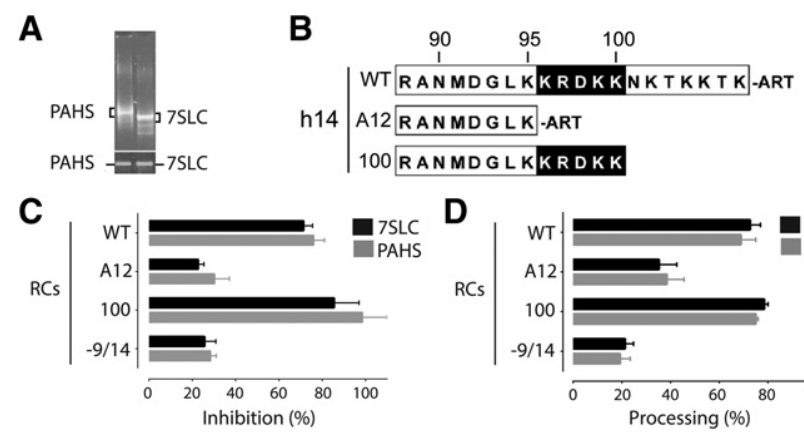

FIGURE 4. Functional analysis of reconstituted SRPs comprising the chimeric human/archaeal SRP RNA PAHS. (A) Ethidium bromidestained gels of 7SLC and PAHS RNAs displayed by native (upper panel) and denaturing (lower panel) PAGE. (B) C-terminal sequences of human SRP14 (h14) proteins used in the functional assays are shown; residue numbers are indicated above. The alanine-rich tail is indicated as ART, for brevity. The sequence known to be essential for elongation arrest activity is shown in white letters on a black background. In h14A12 the basic pentapeptide K96-K100 is removed whereas it is present in h14-100. $(C)$ and $(D)$ Reconstitution reactions were added to translation reactions at a final concentration of $100 \mathrm{nM}$. The translation products were displayed by SDS-PAGE and quantified by phosphor-imager (Supplemental Fig. 4B,C). Elongation arrest activity $(C)$ was determined as the relative inhibition in preprolactin synthesis as compared with cyclin synthesis. The translocation assays contained salt-washed canine microsomes at $0.02 \mathrm{eq} / \mu \mathrm{L}$. The translocation efficiency $(D)$ is determined as the percentage of total preprolactin processed into prolactin (Mary et al. 2010). Standard errors of the mean are shown as SEM $(n \geq 2)$. SRP was reconstituted with either 7SLC (black bars) or PAHS RNA (gray bars) together with recombinant (SRP19, SRP54) and canine (SRP68/72) proteins as well as the SRP9/14 proteins indicated. WT: SRP9/14; -9/14: without SRP9/14. lactin and cyclin mRNAs. The elongation arrest and translocation activities were assayed by determining the relative inhibition of preprolactin synthesis as compared with cyclin synthesis and by quantifying the processing of preprolactin to prolactin in the presence of microsomes. This heterologous system has been extensively used for the characterization of elongation arrest activity (Walter and Blobel 1981; Halic et al. 2004; Mary et al. 2010). The use of wheat-germ lysate is necessary because of the presence of endogenous SRP in the equivalent mammalian translation system. In this system, the elongation arrest activity of reconstituted particles is dependent on the presence of hSRP9/14 (Siegel and Walter 1985). More particularly, a conserved basic pentapeptide located in the C-terminal portion of SRP14 was shown to be required for SRP elongation arrest activity (Fig. 4B; Lakkaraju et al. 2008). PAHS RCs (gray) exhibited similar levels of activity as compared with 7SLC RCs (black) (Fig. 4C,D; Supplemental Table 3). Particularly, we observed that similarly to 7SLC RCs, the presence of h9/14 was required for PAHSRCs to perform elongation arrest activity.

To further confirm that the mechanism of elongation arrest is the same, we tested both RNAs with previously characterized mutated SRP9/14s (Fig. 4D; Lakkaraju et al. 2008; Mary et al. 2010). The h14-A12 protein lacks the residues comprised between the lysine 95 and the alanine tail including the basic pentapeptide.

H14-100 is truncated after the basic pentapeptide. Again, PAHS-RCs behaved similarly to 7SLC-RCs showing that same amino acids were critical for elongation activity (Fig. $4 \mathrm{C})$. The defects in elongation arrest were confirmed in translocation experiments. Particles reconstituted with both RNAs behaved similarly and the translocation efficiencies were reduced in the absence of elongation arrest activity (Fig. 4D).

\section{DISCUSSION}

Using an archaeal $A l u$ RNA, in which the presence of the J1/ $2 / 5$ junction prevents the $180^{\circ}$ rotation of the $5^{\prime}$ relative to the $3^{\prime}$ domain, we have succeeded in determining the structure of the closed, active conformation of the complex. This state was predicted from the crystallographic work on the human Alu domain (Weichenrieder et al. 2000) and confirmed by modeling into the low-resolution cryo-electron microscopy map of the entire mammalian SRP-ribosome complex (Halic et al. 2004). Thus nature may have evolved two ways to stabilize the active closed conformation of the Alu domain RNA. Higher eukaryotes utilize the SRP9/14 heterodimer to clamp together the $5^{\prime}$ and $3^{\prime}$ domains, while archaea (and probably eubacteria with an Alu domain), use an extra helix (H1) which forms a rigid three-way junction, combined with an intricate network of tertiary base-pairing to stabilize the required RNA conformation, possibly also in the absence of protein.

Since the more rigid P. horikoshii Alu RNA is able to functionally replace the equivalent human domain in elongation 
arrest, it is likely that the conformational interchange between open and closed states described for the human Alu domain (Weichenrieder et al. 2001), is not required for this function, but may have another role in eukaryotic systems, where SRP9/ 14 interacts not only with SRP but also with Alu RNA derived from highly repetitive $A l u$ repeats in the genome (Bovia et al. 1995; Hsu et al. 1995). So far, SRP9 or SRP14 homologs have not been identified in archaea (http://rnp.uthscsa.edu/rnp/ SRPDB/SRPDB.html). This could imply either that they are unrecognizable due to sequence divergence or that there are no $A l u$ binding proteins in archaea and the $A l u$ RNA functions as naked $A l u$ RNA. In the absence of a crystal structure of the naked PhAlu RNA it is not known whether it could fold into the same structure without protein. A third possibility is that paralogous Alu RNA binding protein(s) might exist since otherwise it is not easy to understand why the evolutionarily distant archaeal RNA is still able to bind hSRP9/14 in essentially the same manner as human Alu RNA. The only other explanation for this observation is that the RNA structure needs to be conserved in order to be able to interact with highly conserved features of the ribosome.

As observed in the current and previous structures, the SRP14 C-terminal tail that is required for elongation arrest activity extends toward the S-domain when bound to SRP RNA (Fig. 3A). Cross-linking studies in ongoing translation demonstrated that SRP14, and thus the Alu domain, is already in close proximity of the large ribosomal subunit in the absence of a signal sequence in the nascent chain. Upon signal sequence recognition, changes occur at the SRP-ribosome interface and SRP14 is now cross-linked to proteins of the large and the small subunit (Huck et al. 2004). Cryo-electron microscopic (cryo-EM) studies of SRP bound to elongation-arrested ribosomes (Halic et al. 2004, 2006) shows that the Alu domain binds in a cleft between the two subunits, which is the site of interaction of the eukaryotic elongation factor II (eEF2) (Gomez-Lorenzo et al. 2000; Halic et al. 2004) and SRP9/14 contacts three helices of the $18 \mathrm{~S}$ rRNA (Halic et al. 2004). Specifically, residues 57-75 including the functionally essential residues 60, 61, and 64 of SRP9 (Mary et al. 2010) are in close proximity to helices $\mathrm{h} 5$ and $\mathrm{h} 15$ of the small subunit. However, these EM structures have a rather low resolution $(\sim 12 \AA)$ and fail to explain satisfactorily the observed crosslinks with ribosomal proteins of the large subunit and how the essential basic amino acid residues in SRP14 mediate elongation arrest. The new high-resolution structure of the active Alu domain conformation will be valuable in conjunction with a future, improved resolution EM structure of the SRP-ribosome complex in answering these outstanding questions.

\section{DATA DEPOSITION}

Structure factors and coordinates for the complexes of PhAlu134 and PhAlu110 with hSRP9/14 are available in the PDB with codes $4 \mathrm{UYK}$ and $4 \mathrm{UYJ}$, respectively.

\section{SUPPLEMENTAL MATERIAL}

Supplemental material is available for this article.

\section{ACKNOWLEDGMENTS}

We thank the European Commission Directorate-General for Research and Innovation EU FP4 TMR program network SRPNET (FMRX-CT960035) and FP5 Quality of Life program network MEMPROT-NET (QLK3-CT200082) for funding of L.B. and M.A. B. We thank the staff of ESRF and EMBL Joint Structural Biology Group for technical support during ESRF beam time. K.S., C.M., and A.S. acknowledge funding from the Swiss National Science Foundation and the Canton of Geneva.

Received July 6, 2014; accepted September 4, 2014.

\section{REFERENCES}

Birse DE, Kapp U, Strub K, Cusack S, Aberg A. 1997. The crystal structure of the signal recognition particle Alu RNA binding heterodimer, SRP9/14. EMBO J 16: 3757-3766.

Bovia F, Fornallaz M, Leffers H, Strub K. 1995. The SRP9/14 subunit of the signal recognition particle (SRP) is present in more than 20 -fold excess over SRP in primate cells and exists primarily free but also in complex with small cytoplasmic Alu RNAs. Mol Biol Cell 6: 471-484.

Bui N, Wolff N, Cusack S, Strub K. 1997. Mutational analysis of the protein subunits of the signal recognition particle Alu-domain. RNA 3: 748-763.

Cate JH, Gooding AR, Podell E, Zhou K, Golden BL, Szewczak AA, Kundrot CE, Cech TR, Doudna JA. 1996. RNA tertiary structure mediation by adenosine platforms. Science 273: 1696-1699.

Conn GL, Draper DE, Lattman EE, Gittis AG. 1999. Crystal structure of a conserved ribosomal protein-RNA complex. Science 284: 1171-1174.

Doherty EA, Batey RT, Masquida B, Doudna JA. 2001. A universal mode of helix packing in RNA. Nat Struct Biol 8: 339-343.

Gomez-Lorenzo MG, Spahn CM, Agrawal RK, Grassucci RA, Penczek P, Chakraburtty K, Ballesta JP, Lavandera JL, Garcia-Bustos JF, Frank J. 2000. Three-dimensional cryo-electron microscopy localization of EF2 in the Saccharomyces cerevisiae $80 \mathrm{~S}$ ribosome at $17.5 \AA$ resolution. EMBO J 19: 2710-2718.

Halic M, Becker T, Pool MR, Spahn CM, Grassucci RA, Frank J, Beckmann R. 2004. Structure of the signal recognition particle interacting with the elongation-arrested ribosome. Nature 427: 808-814.

Halic M, Gartmann M, Schlenker O, Mielke T, Pool MR, Sinning I, Beckmann R. 2006. Signal recognition particle receptor exposes the ribosomal translocon binding site. Science 312: 745-747.

Hsu K, Chang DY, Maraia RJ. 1995. Human signal recognition particle (SRP) Alu-associated protein also binds Alu interspersed repeat sequence RNAs. Characterization of human SRP9. J Biol Chem 270: 10179-10186.

Huck L, Scherrer A, Terzi L, Johnson AE, Bernstein HD, Cusack S, Weichenrieder O, Strub K. 2004. Conserved tertiary base pairing ensures proper RNA folding and efficient assembly of the signal recognition particle Alu domain. Nucleic Acids Res 32: 4915-4924.

Lakkaraju AK, Mary C, Scherrer A, Johnson AE, Strub K. 2008. SRP keeps polypeptides translocation-competent by slowing translation to match limiting ER-targeting sites. Cell 133: 440-451.

Larsen N, Zwieb C. 1991. SRP-RNA sequence alignment and secondary structure. Nucleic Acids Res 19: 209-215.

Mary C, Scherrer A, Huck L, Lakkaraju AK, Thomas Y, Johnson AE, Strub K. 2010. Residues in SRP9/14 essential for elongation arrest activity of the signal recognition particle define a positively charged functional domain on one side of the protein. RNA 16: 969-979. 


\section{Bousset et al.}

Nissen P, Ippolito JA, Ban N, Moore PB, Steitz TA. 2001. RNA tertiary interactions in the large ribosomal subunit: the A-minor motif. Proc Natl Acad Sci 98: 4899-4903.

Nyathi Y, Wilkinson BM, Pool MR. 2013. Co-translational targeting and translocation of proteins to the endoplasmic reticulum. Biochim Biophys Acta 1833: 2392-2402.

Poritz MA, Siegel V, Hansen W, Walter P. 1988. Small ribonucleoproteins in Schizosaccharomyces pombe and Yarrowia lipolytica homologous to signal recognition particle. Proc Natl Acad Sci 85: 4315-4319.

Saraogi I, Shan SO. 2011. Molecular mechanism of co-translational protein targeting by the signal recognition particle. Traffic 12: 535-542.

Siegel V, Walter P. 1985. Elongation arrest is not a prerequisite for secretory protein translocation across the microsomal membrane. J Cell Biol 100: 1913-1921.

Siegel V, Walter P. 1988. Each of the activities of signal recognition particle (SRP) is contained within a distinct domain: analysis of biochemical mutants of SRP. Cell 52: 39-49.

Strub K, Moss J, Walter P. 1991. Binding sites of the 9- and 14-kilodalton heterodimeric protein subunit of the signal recognition particle (SRP) are contained exclusively in the Alu domain of SRP RNA and contain a sequence motif that is conserved in evolution. Mol Cell Biol 11: 3949-3959.
Thomas Y, Bui N, Strub K. 1997. A truncation in the $14 \mathrm{kDa}$ protein of the signal recognition particle leads to tertiary structure changes in the RNA and abolishes the elongation arrest activity of the particle. Nucleic Acids Res 25: 1920-1929.

Walter P, Blobel G. 1980. Purification of a membrane-associated protein complex required for protein translocation across the endoplasmic reticulum. Proc Natl Acad Sci 77: 7112-7116.

Walter P, Blobel G. 1981. Translocation of proteins across the endoplasmic reticulum III. Signal recognition protein (SRP) causes signal sequence-dependent and site-specific arrest of chain elongation that is released by microsomal membranes. J Cell Biol 91: 557-561.

Weichenrieder O, Wild K, Strub K, Cusack S. 2000. Structure and assembly of the Alu domain of the mammalian signal recognition particle. Nature 408: 167-173.

Weichenrieder O, Stehlin C, Kapp U, Birse DE, Timmins PA, Strub K, Cusack S. 2001. Hierarchical assembly of the Alu domain of the mammalian signal recognition particle. RNA 7: 731-740.

Zwieb C, Bhuiyan S. 2010. Archaea signal recognition particle shows the way. Archaea 2010: 485051.

Zwieb C, van Nues RW, Rosenblad MA, Brown JD, Samuelsson T. 2005. A nomenclature for all signal recognition particle RNAs. RNA 11: $7-13$. 

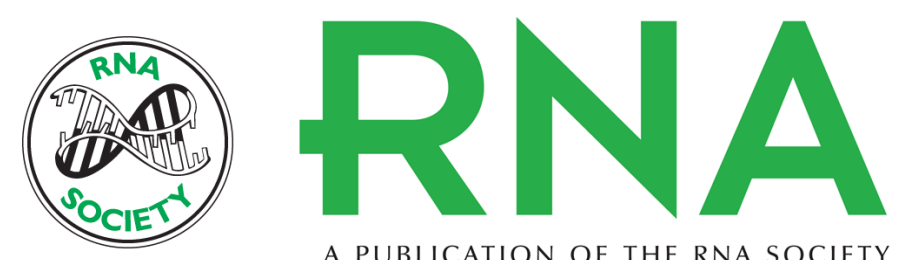

A PUBLICATION OF THE RNA SOCIETY

\section{Crystal structure of a signal recognition particle Alu domain in the elongation arrest conformation}

Luc Bousset, Camille Mary, Mark A. Brooks, et al.

RNA 2014 20: 1955-1962 originally published online October 21, 2014

Access the most recent version at doi:10.1261/rna.047209.114

\section{Supplemental http://rnajournal.cshlp.org/content/suppl/2014/10/06/rna.047209.114.DC1 \\ Material}

References This article cites 28 articles, 17 of which can be accessed free at: http://rnajournal.cshlp.org/content/20/12/1955.full.html\#ref-list-1

Creative This article is distributed exclusively by the RNA Society for the first 12 months after the Commons

License full-issue publication date (see http://rnajournal.cshlp.org/site/misc/terms.xhtml). After 12 months, it is available under a Creative Commons License (Attribution-NonCommercial 4.0 International), as described at http://creativecommons.org/licenses/by-nc/4.0/.
Email Alerting Receive free email alerts when new articles cite this article - sign up in the box at the Service top right corner of the article or click here.

\section{|||||||| Providing Precise Solutions for your research.}

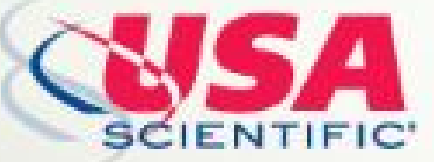

To subscribe to $R N A$ go to:

http://rnajournal.cshlp.org/subscriptions

(C) 2014 Bousset et al.; Published by Cold Spring Harbor Laboratory Press for the RNA Society 